\title{
Reversible lung fibrosis in a 6-year-old girl after long term nitrofurantoin treatment
}

\author{
Lise Fischer Mikkelsen ${ }^{*}$ (iD and Sune Rubak
}

\begin{abstract}
Background: Pulmonary side effects are well known, including lung fibrosis, in elderly patients treated with longterm nitrofurantoin to prevent urinary tract infections and secondary renal injury. However, pulmonary side effects have only been reported rarely in paediatric cases, despite nitrofurantoin being a first line prophylactic treatment of recurrent childhood urinary tract infection.

Case presentations: A 6-year-old girl was admitted to the hospital with dyspnea, general fatigue, loss of appetite and need for nasal oxygen treatment after long-term nitrofurantoin treatment. A computed tomography scan of the chest showed lung fibrosis. A biopsy confirmed this diagnosis. We suspected the fibrosis to be caused by the nitrofurantoin treatment. Thorough examinations reveal no other explanations. Nitrofurantoin was discontinued and the girl was treated with methylprednisolone. After 17 month a new scan and lung function test showed total regression of the lung fibrosis.
\end{abstract}

Conclusions: This case underlines that risk of severe side effects should be taken in to account before initiation of long-term nitrofurantoin treatment in children.

Keywords: Nitrofurantoin, Side effects, Urinary tract infection, Lung fibrosis, Paediatrics

\section{Background}

Nitrofurantoin has been a first line antibiotic choice in prophylactic treatment of childhood urinary tract infection. Pulmonary toxicity causing irreversible pulmonary fibrosis is a well-known side effect of long-term nitrofurantoin treatment and other biological treatments in adults and elderly [1-4]. However, only few cases have been reported of pulmonary affection in children after nitrofurantoin treatment $[5,6]$.

We report a case of a 6-year-old girl who developed dyspnea and interim need for nasal oxygen treatment due to pulmonary fibrosis manifesting after 2 years of nitrofurantoin treatment.

*Correspondence: lisehr@rm.dk

Department of Paediatrics and Adolescent Medicine, Danish Center of Paediatric Pulmonology and Allergology, Aarhus University Hospital, Aarhus N, Denmark

\section{Case presentation}

The 6-year-old girl was admitted to the hospital with dyspnea, general fatigue and loss of appetite developing during approximately 1 month. At admission, oxygen desaturation was $80-90 \%$ before nasal oxygen treatment.

Daily and continuous treatment with oral nitrofurantoin (tablets, $25 \mathrm{mg} /$ day) in combination with solifenacin (tablets) had been initiated 2 years prior to the admission to prevent recurrent urinary tract infections. She presented no other medical history.

Initial blood samples revealed liver affection (p-lactic acid dehydrogenase was $199 \mathrm{U} / \mathrm{L}$ and $\mathrm{p}$-alanintransaminase was $750 \mathrm{U} / \mathrm{L}$ ), but otherwise biochemical parameters were unaffected. Multiple PCR analyses detected no microorganisms in samples from the upper respiratory tract. A computed tomography scan of the chest showed bilateral multilobar parenchymal infiltrates, ground glass opacity, interstitial changes, and enlarged hilar lymph nodes however only discrete signs of lung 
fibrosis with no honeycomb change, subpleural cysts or traction bronchiectasis (Fig. 1). Lung biopsy confirmed suspicion of drug induced lung fibrosis with chronic interstitial inflammation and microscopically diffuse alveolar damage and atypical distribution of the fibrosis involving both the lower and upper lobes bilateral.

On the suspected diagnosis of nitrofurantoin-induced pulmonary fibrosis and due to clinical deterioration including oxygen desaturation, dyspnea, restrictive pattern of lung function, initial treatment with intravenous methylprednisolone $(35 \mathrm{mg} / \mathrm{kg})$ was started at the day of admission. Nitrofurantoin and solifenacin were discontinued. After 3 days, treatment was changed to prednisolone tablets $(20 \mathrm{mg} / \mathrm{kg}$ twice a day).

A bronchoscopy performed after 2 months of treatment due to ongoing respiratory symptomes and revealed structurally normal airways. However, microbiological analyses showed pneumocystis jirovecii in a broncho-alveolar lavage sample taken during the procedure. The infection was successfully treated with tablets of sulfametoxazol and trimetoprim $(400 \mathrm{mg}+80 \mathrm{mg}$ ) three times a day for 3 weeks. It is difficult to be certain about the onset of pneumocystis jirovecii lung infection; however, this infection is most likely a consequence of immunosuppression due to steroid treatment or less likely secondary to the chronic lung changes.

After 2 months, prednisolone was withdrawn over a period of 25 days. However, a following synacthen test showed tertiary adrenal insufficiency and hydrocortisone replacement therapy was initiated.
The girl was followed with frequent consultations and pulmonary function tests. Initial tests showed a restrictive pattern with reduced forced vital capacity (FVC) (69\% of predicted value) and forced expiratory volume in $1 \mathrm{~s}$ (FEV1) (76\% of predicted value), but no bronchial obstruction $\left(\mathrm{FEV}_{1} / \mathrm{FCV}\right.$-ratio unaffected). Following tests showed normalization of all parameters (FVC was $103 \%$ and FEV1 was $101 \%$ of predicted value) (Fig. 2). 17 months after the first admission, the girl performed a spirometry test showing normal pulmonary function and a high-rate computed tomography scan showed total regression of the pulmonary fibrosis.

\section{Discussion and conclusions}

Nitrofurantoin is an antibiotic medicament often used in the treatment of recurrent urinary tract infections as the urinary excretion rate is high. Recent studies have questioned the efficacy of antibiotics in the prevention of recurrent urinary tract infections and secondary renal injury in children [7]. In a recent paper in the Lancet, the authors concluded that "a trial using antimicrobial prophylaxis in children with several recurrent episodes of acute pyelonephritis is warranted" [8].

In Denmark, the national paediatric society recently changed the national clinical guideline: Prophylactic antibiotics should only be prescribed by specialists and after treatment of relevant risk factors [9]. The recommendation is trimethoprim ( $2 \mathrm{mg} / \mathrm{kg}$ once a day) as first line treatment of recurrent upper urinary tract infection in children (amoxicillin if the child is younger than 6 weeks). Second choice is nitrofurantoin, demanding
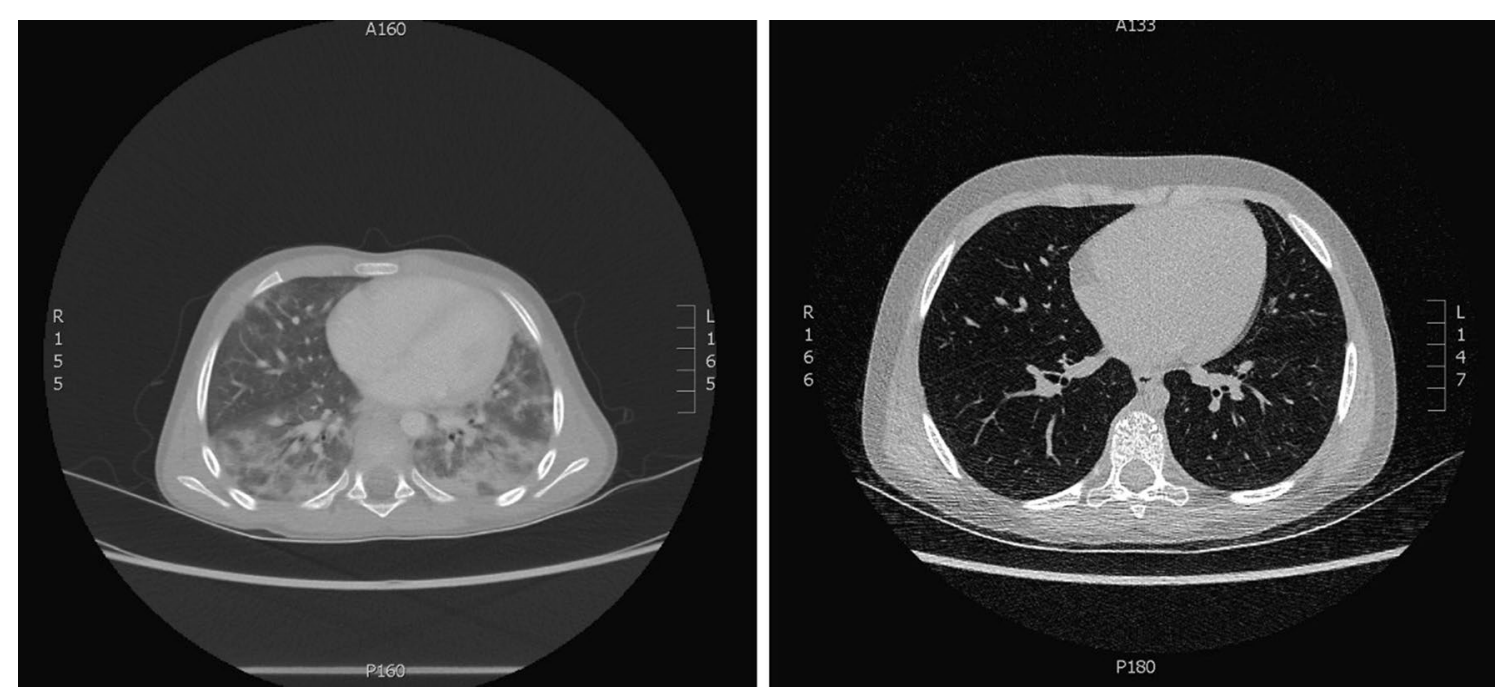

Fig. 1 A computed tomography scan of the chest for the time of admission (left) showing bilateral multilobar parenchymal infiltrates, ground glass opacity, interstitial changes, and enlarged hilar lymph nodes however only discrete signs of lung fibrosis with no honeycomb change. After 8 months post high-dose methylprednisolone treatment (right) $\mathrm{CT}$ showing complete regression of lung findings 


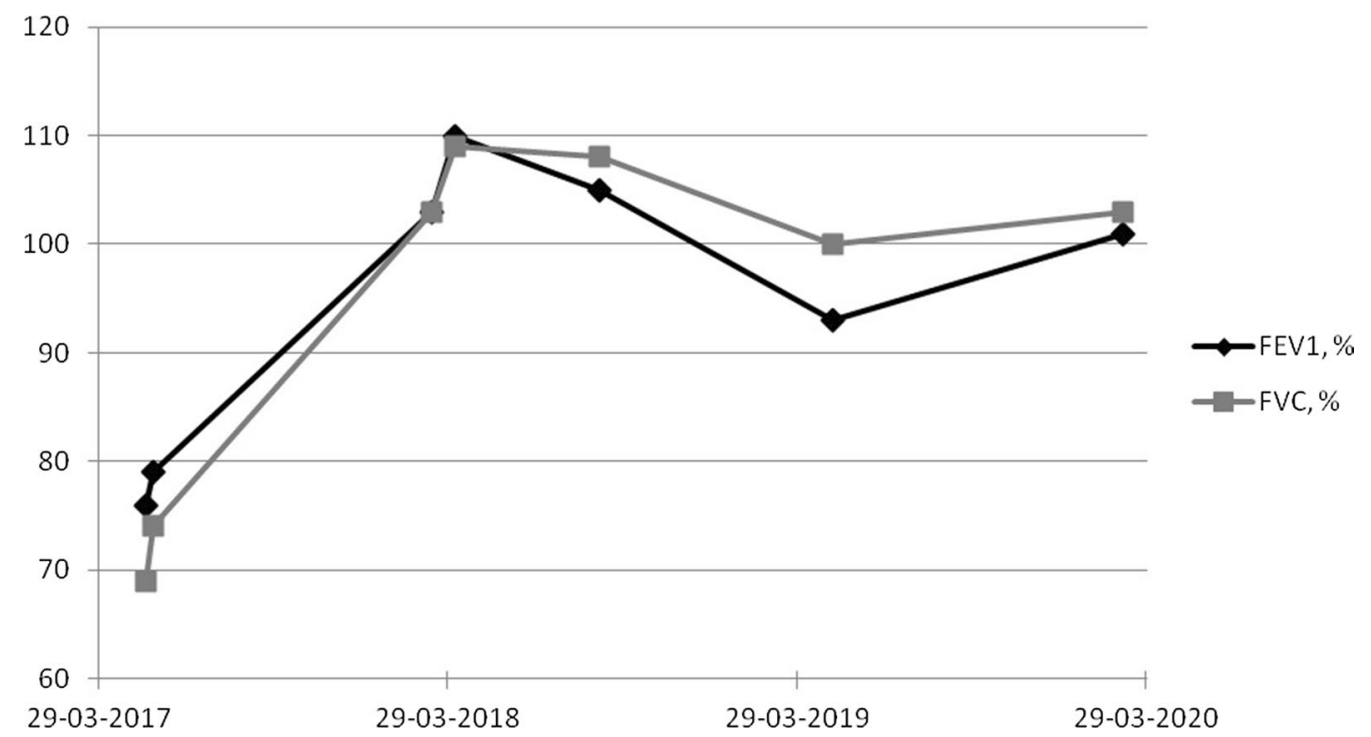

Fig. 2 Progression of lung function over time, FVC and FEV1 in percent of predicted value. Diagnosis of lung fibrosis and discontinuation of nitrofurantoin and initiation of treatment with glucocorticoids at 29/3 2017

regular anamnestic screening of pulmonary symptoms including lung function testing.

The American Academy of Pediatrics (clinical guidelines, latest reaffirmation in 2016) recommends "prompt" initiation of antibiotic treatment in case of symptoms rather than prophylactic antibiotic [10] of recurrent urinary tract infection. Furthermore, the committee emphasizes the importance of treating bowl/bladder dysfunctions, as this is a major, but disregarded, risk factor for recurrent urinary tract infections.

Different pathophysiological mechanisms have been suggested to cause the pulmonary toxitcity of long-term nitrofurantoin treatment. One leading theory is that oxidative stress by the production of free radicals might injure the lung tissue, as nitrofurantoin in its active form is highly reactive. In vivo studies showed reduced injury in tissue incubated with nitrofurantoin in combination with antioxidants compared to nitrofurantoin alone. Hypersensitivity to nitrofurantoin and thereby cytokineinitiated inflammation is another possible explanation. However, hypersensitivity is more likely to cause acute reactions after short-time nitrofurantoin treatment [11].

In this case, a 6-year-old girl developed pulmonary fibrosis after 2 years of nitrofurantoin treatment preventing recurrent urinary tract infections. Thorough clinical examinations and paraclinical testing revealed no infection or other causes of the fibrosis at the time of admission. Discontinuation of nitrofurantoin and treatment of high-dose steroids resulted in full regression of the fibrosis and normalization of pulmonary symptoms and lung function. We conclude that the most probable cause to reversible lung fibrosis was the pulmonary toxicity of treatment with long-term nitrofurantoin. Whether or not the pneumocystis jirovecii infection verified in the lungs 2 months later had been ongoing for longer time is unknown, however if present at baseline and during steroid treatment it would have been expected to be associated with worsening of lung function.

This case shows that the well-known side effect of long-term nitrofurantoin treatment in elderly also may be a rare paediatric risk factor. Clinicians should consider alternative options when planning prophylactic treatment of recurrent urinary tract infections in children.

\section{Abbreviations}

FVC: Forced vital capacity; FEV1: Forced expiratory volume in $1 \mathrm{~s}$.

\section{Acknowledgements}

Not applicable.

\section{Authors' contributions}

SR was main senior clinician treating the patient. LFM wrote the manuscript as primary author. Both authors have participated in the preparation and writing process and have approved the submitted version.

Funding

None.

Availability of data and materials

Not applicable.

Ethics approval and consent to participate Not applicable. 


\section{Consent for publications}

Written informed consent was obtained from the patient's legal guardian(s) for publication of this case report and any accompanying images. A copy of the written consent is available for review by the Editor-in-Chief of this journal.

\section{Competing interests}

The authors declare that they have no competing interests.

Received: 12 September 2020 Accepted: 19 November 2020

Published online: 26 November 2020

\section{References}

1. Almeida P, Seixas E, Pinheiro B, Ferreira P, Araujo A. Consider nitrofurantoin as a cause of lung injury. Eur J Case Rep Intern Med. 2019;6(11):001295.

2. Kabbara WK, Kordahi MC. Nitrofurantoin-induced pulmonary toxicity: a case report and review of the literature. J Infect Public Health. 2015;8(4):309-13.

3. Madani, et al. Nitrofurantoin-induced disease and prophylaxis of urinary tract infections. Prim Care Respir J. 2012;21(3):337-41.

4. Karampitsakos, et al. Biologic treatments in interstitial lung diseases. Front Med. 2019. https://doi.org/10.3389/fmed.2019.00041.
5. Karpman E, Kurzrock EA. Adverse reactions of nitrofurantoin, trimethoprim and sulfanethoxazole in children. J Urol. 2004;172(2):448-53.

6. Hage P, El Hajje MJ. Nitrofurantoin-induced desquamative interstitial pneumonitis in a 7-year-old child. Pediatr Infect Dis J. 2011;30(4):363.

7. Williams $\mathrm{G}$, Craig JC. Long-term antibiotics for preventing recurrent urinary tract infection in children. Cochrane Database Syst Rev. 2019:4:CD001534

8. Tullus K, Shaikh N. Urinary Tract Infections in Children. Lancet 2020 May 23:395(10237.

9. Danish Pediatric Society Guidelines. http://www.paediatri.dk/nefrourolo gi-vejl. 2020.

10. Subcommittee on Urinary Tract Infection Reaffirmation of AAP Clinical Practice Guideline. The diagnosis and management of the initial urinary tract infection in febrile infants and young children 2-24 months of age. Pediatrics. 2016;138(6):20163026.

11. Syed H, Bachuwa G, Upadahyaa S, Abed F. Nitrofurantoin-induced interstitial pneumonitis: albeit rare, should not be missed. BMJ Case Rep. 2016: bcr2015213967.

\section{Publisher's Note}

Springer Nature remains neutral with regard to jurisdictional claims in published maps and institutional affiliations.
Ready to submit your research? Choose BMC and benefit from:

- fast, convenient online submission

- thorough peer review by experienced researchers in your field

- rapid publication on acceptance

- support for research data, including large and complex data types

- gold Open Access which fosters wider collaboration and increased citations

- maximum visibility for your research: over 100M website views per year

At $\mathrm{BMC}$, research is always in progress.

Learn more biomedcentral.com/submissions 